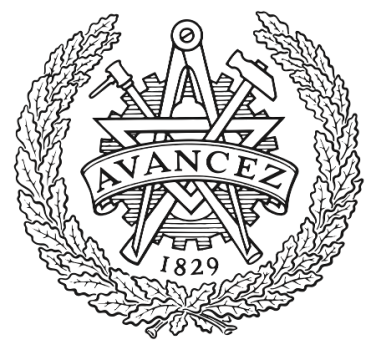

CHALMERS

UNIVERSITY OF TECHNOLOGY

\title{
Strong Electron Localization in Tin Halide Perovskites
}

Downloaded from: https://research.chalmers.se, 2023-04-26 13:07 UTC

Citation for the original published paper (version of record):

Ouhbi, H., Ambrosio, F., De Angelis, F. et al (2021). Strong Electron Localization in Tin Halide

Perovskites. Journal of Physical Chemistry Letters, 12(22): 5339-5343.

http://dx.doi.org/10.1021/acs.jpclett.1c01326

N.B. When citing this work, cite the original published paper. 


\title{
Strong Electron Localization in Tin Halide Perovskites
}

\author{
Hassan Ouhbi, Francesco Ambrosio, Filippo De Angelis, and Julia Wiktor*
}

Cite This: J. Phys. Chem. Lett. 2021, 12, 5339-5343

Read Online

ABSTRACT: Tin halide perovskites (THPs) have been established as a lower-toxicity alternative to lead halide perovskites. In spite of the increasing interest, the behavior of photoexcited charges has not been well understood in this class of materials. We here investigate the behavior of excess electrons in a series of tin halide perovskites by employing advanced electronic-structure calculations. We first focus on $\mathrm{CsSnBr}_{3}$ and show that electron localization is favorable in this compound and that bipolaronic states are the most stable form of self-trapped electrons. We then extend the analysis to $\mathrm{CsSnI}_{3}$, $\mathrm{CsSnCl}_{3}, \mathrm{MASnBr}_{3}, \mathrm{FASnBr}_{3}$, and $\mathrm{DMASnBr}_{3}$ and show that electron bipolarons are stable in all these compounds, thus indicating that strong electron localization is recurrent in THPs.

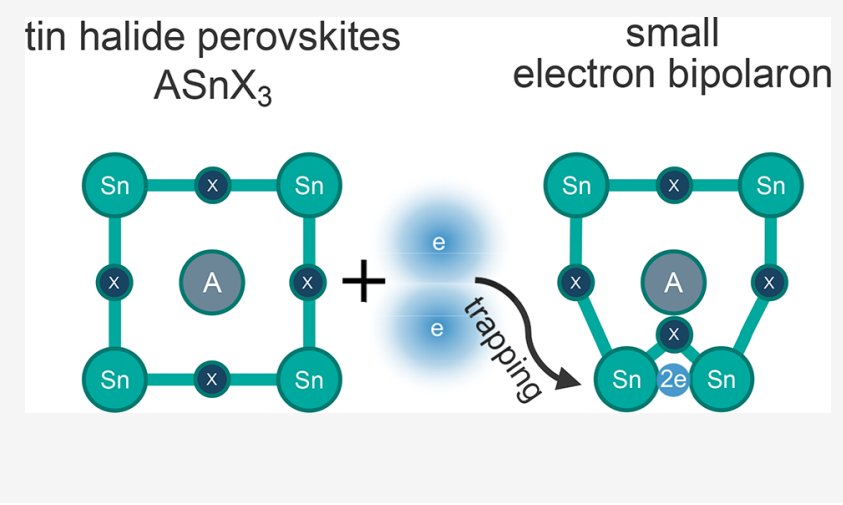

Colar cells based on metal halide perovskites show $\checkmark$ promising photovoltaic properties with power conversion efficiencies improving rapidly over the past few years and now exceeding 25\%. ${ }^{1}$ The highest efficiencies to date have been obtained with compounds containing lead (lead halide perovskites, LHPs), a toxic element. ${ }^{2-5}$ Tin halide perovskites (THPs) exhibit a lower toxicity ${ }^{6}$ and are considered as one of the most promising replacement of LHPs because of their high absorption coefficients, long lifetimes of photogenerated charges, and low impact of defects. ${ }^{7-10}$ The main issue hindering the successful use of THPs is related to their stability, which is hampered by charge-trapping processes. In fact, It has been shown that THPs have stronger tendency to localize extra charges. ${ }^{11}$ This characteristic, in conjunction with the facile oxidation of surface $\mathrm{Sn}$ (II) to $\mathrm{Sn}(\mathrm{IV}),{ }^{12}$ leading to the formation of secondary phases, is at the root of the poor thermodynamic stability of THPs. Such a drawback, intrinsic to tin chemistry, might be circumvented by either deploying surface passivation strategies ${ }^{10}$ or by alloying tin with lead ${ }^{13}$ in lead-alleviated perovskites. However, these strategies address mainly hole-trapping processes while not accounting for electron localization, which may play a major role in THPs, as their band edges are generally closer to the vacuum level with respect to LHPs. ${ }^{10}$ Therefore, a detailed analysis of the electron-trapping phenomena in LHPs is paramount for development of their electronic properties toward the realization of highly efficient devices for photovoltaics.

In the present work, we study the behavior of excess electrons in THPs through advanced ab initio calculations. We first consider single- and double-electron polarons in "polymorphous"14 cubic structures of $\mathrm{CsSnBr}_{3}$ and then extend the analysis to other THPs. We show that double polarons (bipolarons), associated with the formation of $\mathrm{Sn}-\mathrm{Sn}$ bonds and leading to strong electron-trapping, are stable in all the studied THPs, thus indicating a common behavior for this class of materials.

We first focus on $\mathrm{CsSnBr}_{3}$ as a representative tin-based halide perovskite. This compound adopts a cubic structure at room temperature. ${ }^{15}$ Modeling the cubic structure of halide perovskites is counterintuitively a challenging issue. The effectively cubic structure of a material could suggest that a highly symmetric model can be used to describe the structure. However, because this is a high-temperature phase, the symmetric structure is preserved only on average, while locally strong distortions are present. These instantaneous distortions have been assessed for instance through molecular dynamics ${ }^{16}$ and have been shown to affect the electronic structure of the material significantly. The problem also manifests itself when supercells of the symmetric cubic phase are constructed and relaxed at $0 \mathrm{~K}$. Zhao et al. has shown that in such a supercell a distribution of different low-symmetry local motifs can be observed, called "polymorphous networks". ${ }^{14}$ This means that a single structure might not be enough to study structural and electronic properties of cubic perovskites.

In the present study we use the $\mathrm{CP} 2 \mathrm{~K}$ package p $^{17,18}$ to generate the various polymorphous structures, both neutral and charged systems. The calculations are performed at the hybrid functional level, using the $\operatorname{PBEO}(\alpha) \cdot{ }^{19}$ In the $\operatorname{PBE0}(\alpha)$ calculations for $\mathrm{CsSnBr}_{3}$ we set the $\alpha$ parameter to 0.26 , as

Received: April 23, 2021

Accepted: May 27, 2021

Published: June 1, 2021 
determined in ref 20 based on the generalized Koopmans' theorem. We consider a $4 \times 4 \times 4$ cubic supercell containing 320 atoms, with the experimental lattice parameter of $a=$ $5.8043 \AA^{15}$ Additional computational details are given in the Supporting Information.

To overcome the issue of the instability of the perfectly cubic structure at $0 \mathrm{~K},{ }^{14}$ we construct 10 structures with different initial random atomic displacements (up to $0.15 \AA$ ). This number of models is enough to achieve good statistics, in accord with ref 14 showing that the electronic structure varies only slightly between the structures. The structures are first fully relaxed before introducing extra charges. To find the configurations of the single-electron polaron, we add one extra charge to each of the 10 neutral structures, beginning the relaxation with $\operatorname{PBE0}(\alpha=0.5)$ to overcome possible energy barriers for charge localization. Once the charge is localized, we continue the relaxation with $\operatorname{PBE} 0(\alpha=0.26)$. To form doubleelectron polarons, in each neutral structure we identify the pair of $\mathrm{Sn}$ atoms with the smallest distance. We then reduce the $\mathrm{Sn}-\mathrm{Sn}$ separation to about $3.2 \AA$, while displacing the middle $\mathrm{Br}$, based on bipolaronic geometries of $\mathrm{CsPbBr}_{3}$ found in ref 21 . Finally, we relax the geometry completely with $\operatorname{PBE} 0(\alpha=$ $0.26)$.

Because the $\mathrm{CP} 2 \mathrm{~K}$ package does not allow taking into account the effect of spin-orbit coupling (SOC), which can be significant in tin-based perovskites, we perform additional calculations in the Vienna ab initio package (VASP). ${ }^{22,23}$ The calculations are carried out on top of the final geometries, as achieved with the $\mathrm{CP} 2 \mathrm{~K}$ package. Additional details related to these calculations are given in the Supporting Information. From VASP calculations we extract the difference between formation energies of polarons with and without SOC, at the
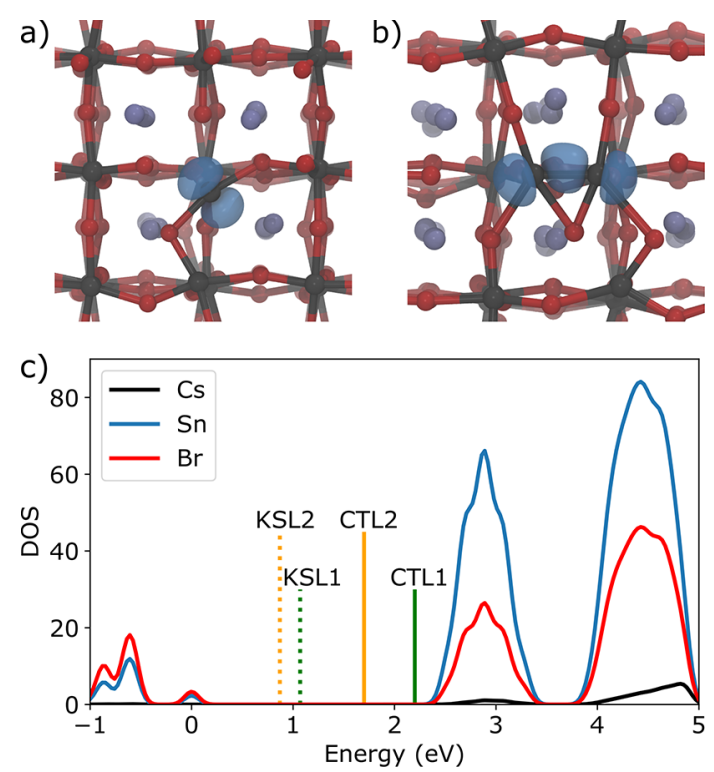

Figure 1. Representative isodensities (in blue, at $20 \%$ of the maximum value) of the (a) single- and (b) double-electron polarons in $\mathrm{CsSnBr}_{3}$. Cs, $\mathrm{Sn}$, and $\mathrm{Br}$ atoms are shown in violet, gray, and red, respectively. (c) Partial densities of states of $\mathrm{CsSnBr}_{3}$, aligned with the Kohn-Sham and charge transition levels. KSL1 and CTL1 correspond to the single-electron polarons and KSL2 and CTL2 to the bipolaron.
PBE level. The energy differences are then added to the formation energies found with the $\mathrm{CP} 2 \mathrm{~K}$ package, following

$$
E_{\mathrm{b}}^{\mathrm{SOC}}=E_{\mathrm{b}}^{\mathrm{noSOC}, \mathrm{CP} 2 \mathrm{~K}}+\Delta^{\text {SOC,VASP }}
$$

with

$$
\Delta^{\text {SOC,VASP }}=E_{\mathrm{b}}^{\text {SOC,VASP }}-E_{\mathrm{b}}^{\text {noSOC,VASP }}
$$

To verify the validity of including the SOC effect calculated at the PBE level on fixed geometries obtained without SOC in $\mathrm{CP} 2 \mathrm{~K}$, we also perform one calculation using the full PBE0+SOC method in VASP, including the geometry optimization. For the bipolaron in $\mathrm{CsSnBr}_{3}$, the formation energies obtained with the two approaches differ by only 0.03 $\mathrm{eV}$.

We now focus on single-electron polarons in $\mathrm{CsSnBr}_{3}$. In all studied structures, we observe electron localization related to the elongation of three $\mathrm{Sn}-\mathrm{Br}$ bonds. The polaronic configuration is highly asymmetrical, with the elongated bonds measuring on average $3.37,3.71$, and $4.13 \AA$, compared to about $2.91 \AA$ in the neutral structures. A representative polaronic configuration is shown in Figure 1a. The formation energies of single-electron polarons are calculated as follows:

$$
E_{\mathrm{b}}=E_{-1}[\mathrm{pol}]-E_{0}[\text { pristine }]-\epsilon_{\mathrm{c}}
$$

where $E_{-1}[\mathrm{pol}]$ is the total energy of the relaxed supercell containing the single-electron polaron, $E_{0}$ [pristine] the energy of pristine $\mathrm{CsSnBr}$, and $\epsilon_{\mathrm{c}}$ the position of the conduction band minimum (CBM). We here neglect the electrostatic finite-size correction because of the high dielectric constant $\left(68.3^{15}\right)$ of $\mathrm{CsSnBr}_{3}$. We note that in this notation, a negative formation energy indicates that the polaronic state is energetically favorable.

Table 1. Space Groups and Lattice Parameters of the Considered Tin-Based Halide Perovskites ${ }^{a}$

$\begin{array}{lcccccc} & \text { space group } & a(\AA) & b(\AA) & c(\AA) & \alpha & E_{\text {gap }}^{\text {exp }}(\mathrm{eV}) \\ \mathrm{CsSnI}_{3} & P n m a^{b} & 8.69 & 12.38 & 8.64 & 0.23 & 1.3^{b} \\ \mathrm{CsSnBr} & P m \overline{3} m^{c} & 5.80 & 5.80 & 5.80 & 0.26 & 1.8^{c} \\ \mathrm{CsSnCl}_{3} & P m \overline{3} m^{d} & 5.56 & 5.56 & 5.56 & 0.35 & 2.8^{d} \\ \mathrm{MASnBr}_{3} & P m \overline{3} m^{e} & 5.91 & 5.91 & 5.91 & 0.20 & 2.0^{e} \\ \mathrm{FASnBr}_{3} & P m \overline{3} m^{f} & 6.00 & 6.00 & 6.00 & 0.23 & 2.4^{f} \\ \mathrm{DMASnBr}_{3} & P b c a^{g} & 6.15 & 6.08 & 6.08 & 0.18 & 2.9^{g}\end{array}$

${ }^{a} \alpha$ is the fraction of Fock exchange incorporated in the $\operatorname{PBEO}(\alpha)$ functional. ${ }^{b}$ Experimental data come from ref $7 .{ }^{c}$ Experimental data come from refs 15 and 29. ${ }^{d}$ Experimental data come from refs 33 and 26. ${ }^{e}$ Experimental data come from ref $34 .{ }^{f}$ Experimental data come from ref $35 .{ }^{g}$ Experimental data come from ref 36 .

Table 2. Formation Energies (per Charge) of the Electron Bipolarons in Various Tin-Based Halide Perovskites ${ }^{a}$

$\begin{array}{lccc} & E_{\mathrm{b}}(\mathrm{eV}) \text { without SOC } & E_{\mathrm{b}}(\mathrm{eV}) \text { with SOC } & d_{\mathrm{Sn}-\mathrm{Sn}}(\AA) \\ \mathrm{CsSnI}_{3} & -0.35 & -0.12 & 3.12 \\ \mathrm{CsSnBr}_{3} & -0.80 & -0.54 & 3.07 \\ \mathrm{CsSnCl}_{3} & -1.43 & -1.23 & 3.03 \\ \mathrm{CsSnBr}_{3} & -0.80 & -0.54 & 3.07 \\ \mathrm{MASnBr}_{3} & -0.46 & -0.32 & 3.13 \\ \mathrm{FASnBr}_{3} & -0.68 & -0.46 & 3.09 \\ \mathrm{DMASnBr}_{3} & -0.51 & -0.36 & 3.15\end{array}$

${ }^{a}$ Values before and after including SOC are given. The $\mathrm{Sn}-\mathrm{Sn}$ bond length $\left(d_{\mathrm{Sn}-\mathrm{Sn}}(\AA)\right)$ is also given. 

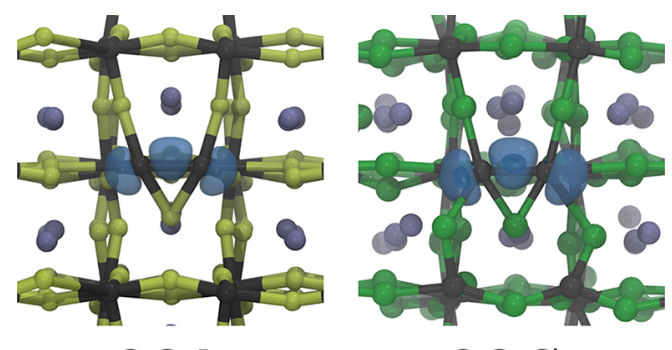

$\mathrm{CsSnI}_{3}$

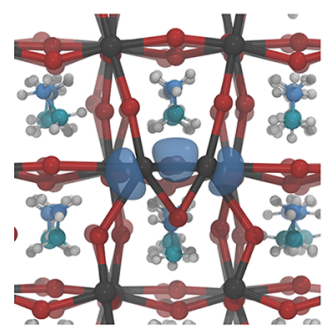

$\mathrm{MASnBr}_{3}$

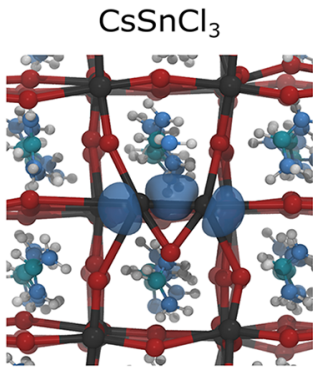

$\mathrm{FASnBr}_{3}$

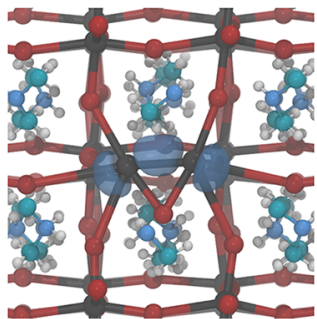

$\mathrm{DMASnBr}_{3}$

Figure 2. Isodensities (in blue, at $20 \%$ of the maximum value) of the bipolaronic states in $\mathrm{CsSnI}_{3}, \mathrm{CsSnCl}_{3}, \mathrm{MASnBr}_{3}, \mathrm{FASnBr}_{3}$, and $\mathrm{DMASnBr}_{3}$.

Without SOC effects, we find the formation energy of the single-electron polaron in $\mathrm{CsSnBr}$ to amount to, on average, $-0.30 \pm 0.05 \mathrm{eV}$, suggesting significant stability of the polaronic state. The incertitude is calculated as the mean absolute error (MAE) for the 10 structures. The singleelectron polaron is associated with a one-particle Kohn-Sham level (KSL) found at $1.43 \mathrm{eV}$ below the conduction band minimum, as shown in Figure 1b. Upon inclusion of the SOC effect, the formation energy is increased by about $0.24 \mathrm{eV}$, leading to the final value of $-0.06 \pm 0.05 \mathrm{eV}$, suggesting only a weak stability of single-electron polarons in $\mathrm{CsSnBr}_{3}$. We note that in some of the polymorphous models the single-electron polarons are particularly stable (with the lowest value of the formation energy of $-0.17 \mathrm{eV}$ ). This suggests that the charge localization in $\mathrm{CsSnBr}_{3}$ can be dynamically stabilized by favorable local distortions, similarly to what was observed in $\mathrm{CsPbBr}_{3}{ }^{21}$

Next, we focus on double-electron polarons. In $\mathrm{CsPbBr}_{3}$, various bipolaronic states were identified, in which two electrons localized between neighboring metal atoms. We find similar configurations in $\mathrm{CsSnBr}_{3}$, one of which is given in Figure 1. In all 10 considered structures, a bond between two $\mathrm{Sn}$ atoms of on average $3.07 \AA$ is formed, while the middle $\mathrm{Br}$ atom is strongly shifted in the direction perpendicular to the $\mathrm{Sn}-\mathrm{Sn}$ bond. We note that the bipolaronic configuration can also be considered as a complex of a $\mathrm{Br}$ interstitial $\left(\mathrm{I}_{\mathrm{Br}}^{-}\right)$and a $\mathrm{Br}$ vacancy $\left(\mathrm{V}_{\mathrm{Br}}^{-}\right)$, both in a negative charge state, because one $\mathrm{Br}$ atom leaves its initial position in the perovskite lattice and shifts toward the interstitial site. This corresponds to an excited state of a Frenkel pair, which is formally a complex of $\mathrm{V}_{\mathrm{Br}}^{+}$and $\mathrm{I}_{\mathrm{Br}}^{-}$

For the bipolaronic configurations we calculate the formation energy per charge as

$$
E_{\mathrm{b}}=\frac{E_{-2}[\text { bipol }]-E_{0}[\text { pristine }]-2 \epsilon_{\mathrm{c}}}{2}
$$

where $E_{-2}[$ bipol $]$ is the total energy of the relaxed supercell containing the bipolaron, and the other quantities are defined as in eq 3. We find that without SOC, the total energy of the bipolaronic state is on average $1.59 \pm 0.07 \mathrm{eV}$ lower than that of a system with two delocalized electrons. This corresponds to the formation energy of $-0.80 \pm 0.03 \mathrm{eV}$ per electron, significantly lower than the $E_{\mathrm{b}}$ of the single polaron $(-0.30 \pm$ $0.05 \mathrm{eV})$. The alignment between the charge transition levels corresponding to the single- and double-electron polaron (without SOC) is also shown in Figure 1c), where we also plot the partial densities of states of $\mathrm{CsSnBr}_{3}$. We find that both the one-particle Kohn-Sham level (KSL) and the charge transition level (CTL) of the bipolaron lies deeper within the band gap. After the inclusion of the SOC effect, the formation energy of the bipolaron is increased by about 0.25 $\mathrm{eV}$, to $-0.54 \pm 0.03 \mathrm{eV}$ per electron. This large magnitude of the formation energy suggests that the electrons in $\mathrm{CsSnBr}_{3}$ are strongly trapped as bipolarons. We note that electron localization is much more favorable in $\mathrm{CsSnBr}_{3}$ than in $\mathrm{CsPbBr}$, where the bipolaronic states were found to be unstable at $0 \mathrm{~K} . .^{21}$ This is related to the higher position of the conduction band in the lead-based perovskites, ${ }^{10}$ which favors electron trapping. At the same time, the high position of the valence band in tin-based perovskites disfavors hole trapping in the material. We note that we have considered the possibility of hole trapping in $\mathrm{CsSnBr}_{3}$ through the formation of both $\mathrm{Sn}(\mathrm{III})$ and $\mathrm{Sn}(\mathrm{IV})$ ions, $\mathrm{Br}-\mathrm{Br}$ dimers, or bromine Frenkel defects, but we have found no stable localized states associated with extra holes, in line with previous studies. ${ }^{10,13}$

We also note that the low stability of the single polaronic states may significantly limit the lifetime of the single-polaron state, in turn reducing the probability of capture of a second electron to form the bipolaron. We therefore surmise that electron bipolarons will be mainly formed under high irradiation regimes, i.e. upon establishment of a significant carrier density in the perovskite. As previously found for both lead- and tin-halide perovskites, charge trapping at defects at surfaces and grain boundaries may stabilize singly trapped charges, ${ }^{12,13,24}$ possibly leading to a preferential channel for bipolaron formation.

Because halide perovskites can adapt different structures, we next assess the effect of the phase on polaron stability. This is done by making a comparison with $\mathrm{CsSnBr}_{3}$ in the orthorhombic structure. In this case we consider a $2 \times 2 \times$ 2 repetition of the unit cell with experimental lattice parameters $a=8.1965, b=11.583$, and $c=8.0243 \AA$, ${ }^{15}$ containing 160 atoms. For the orthorhombic phase, differently from the cubic structure, only one model is needed. For the single-electron polaron we find the formation energy of -0.25 $\mathrm{eV}$ before including SOC effects. Once SOC is included, this value is increased to $-0.01 \mathrm{eV}$. In the case of the bipolaron, without SOC we find the formation energy of $-0.60 \mathrm{eV}$ per electron. When relativistic effects are included, the stability of the bipolaron is reduced and the formation energy amounts to $-0.36 \mathrm{eV}$. This is by about $0.18 \mathrm{eV}$ higher than the value found in the cubic phase $(-0.54 \mathrm{eV})$ suggesting that the polaronic 
states are less stable in the orthorhombic structure of $\mathrm{CsSnBr}_{3}$. In both phases we find that the bipolaronic states are much more stable than the single-electron polarons; therefore, we focus only on this type of localization in the rest of our study.

We now extend our analysis to other compounds, and we verify if the strong electron trapping in the bipolaronic state is a more general phenomenon of THPs. We consider $\mathrm{CsSnI}_{3}$ and $\mathrm{CsSnCl}_{3}$ to assess the effect of the halogen atoms and $\mathrm{MASnBr}_{3}, \mathrm{FASnBr}_{3}$, and $\mathrm{DMASnBr}_{3}$ to evaluate that of the cation. In Table 1 we summarize the structural properties of the considered compounds and the $\alpha$ parameters used in the PBE0 functional. The values are taken from previous studies either based on the generalized Koopmans theorem ${ }^{20}$ or chosen to reproduce the experimental band gap of the material (reference values given in Table 1). We note that in the cases of $\mathrm{CsSnCl}_{3}, \mathrm{MASnBr}_{3}$, and $\mathrm{FASnBr}_{3}$ which are cubic, we take only one polymorphous model, because in $\mathrm{CsSnBr}_{3}$ we observed a small variation of the bipolaron formation energy between the considered models. The formation energies of the bipolarons are given in Table 2, in which we report both the values neglecting and including SOC. The trend within the set of compounds containing Cs can be directly correlated with the size of the band gap, with the smallest bipolaron stability found in $\mathrm{CsSnI}_{3}$ (experimental band gap of $1.3 \mathrm{eV}^{25}$ ) and the largest in $\mathrm{CsSnCl}_{3}$ (experimental band gap of $2.8 \mathrm{eV}^{26}$ ). The trend can also be observed from the alignment of polaronic levels with the densities of states of the various perovskites shown in Figure S1. The change of cation has a less straightforward effect on the bipolaron formation energy, in line with what was observed in lead halide perovskites. ${ }^{27}$ For instance, even though the band gap of $\mathrm{DMASnBr}_{3}$ is significantly larger than that of $\mathrm{CsSnBr}_{3}\left(2.9 \mathrm{eV}^{28}\right.$ compared to $\left.1.8 \mathrm{eV}^{29}\right)$, the bipolaron is less stable in the former material. This can be explained based on a larger volume per unitary formula, increasing the deformation cost of bringing two Sn atoms closer, thus suggesting another handle to tune the electronic properties of THPs. Nevertheless, we observe that the bipolarons are stable in all considered tin-based halide perovskites. As a consequence, we conclude that in general the excess electrons in THPs can be trapped and therefore their mobilities limited, which could be detrimental to the performance of optoelectronic devices. On the other hand, charge localization could be beneficial, because polaron formation extends charge carrier lifetimes by reducing the overlap between hole and electron wave functions. ${ }^{30}$ The polaron formation has been shown to reduce both monomolecular $^{31}$ and bimolecular ${ }^{32}$ recombination rates in lead halide perovskites. However, the aforementioned observations have been made for more delocalized polarons in $\mathrm{MAPbI}_{3}$, and the effect of small polaron formation in THPs on the charge carrier lifetimes should be further examined.

In conclusion, we studied the behavior of excess electrons in tin halide perovskites using hybrid density functional theory. We first focused on cubic CsSnBr 3 , in which we studied both single- and double-electron polarons. We considered a set of "polymorphous" cubic models to overcome the problems of instability of the cubic perovskite phase at $0 \mathrm{~K}$. We observed that while spin-orbit coupling significantly reduces the stability of polarons in $\mathrm{CsSnBr}_{3}$, they still represent the favorable form of excess electrons. Having found that the bipolaronic states are more stable than two isolated singleelectron polarons, we extended our study to other THPs, namely to $\mathrm{CsSnI}_{3}, \mathrm{CsSnCl}_{3}, \mathrm{MASnBr}_{3}, \mathrm{FASnBr}_{3}$, and
$\mathrm{DMASnBr}_{3}$ (Figure 2), in order to assess the effect of both the halogen and the A-site cation. We observed that bipolaronic states are energetically favorable in all these compounds and can lead to strong electron trapping and reduced mobility of charge carriers. Halogen substitution has been found to induce a straightforward effect on bipolaron stabilization, which was found to increase from I to $\mathrm{Cl}$, following the higher band gap of the material. In contrast, the effect of the A-site cation is less obvious. Because of the larger variation in the volume associated with the change in the A-site cation, the gap-polaron correlation is not preserved in this case. In fact, less compact THPs may entail a larger deformation cost for the formation of the $\mathrm{Sn}-\mathrm{Sn}$, thus lowering the stability of the bipolaron. Overall, our results demonstrate that strong electron trapping is recurrent in THPs and may limit their application in optoelectronic devices.

\section{ASSOCIATED CONTENT}

\section{Supporting Information}

The Supporting Information is available free of charge at https://pubs.acs.org/doi/10.1021/acs.jpclett.1c01326.

Computational details, assessment of finite-size corrections, justification of the parametrization of the hybrid density functionals, and densities of states of all considered tin halide perovskites with the Kohn-Sham and charge transition levels corresponding to the bipolaronic states (PDF)

\section{AUTHOR INFORMATION}

\section{Corresponding Author}

Julia Wiktor - Department of Physics, Chalmers University of Technology, Gothenburg, Sweden; O orcid.org/0000-00033395-1104; Email: julia.wiktor@chalmers.se

\section{Authors}

Hassan Ouhbi - Department of Physics, Chalmers University of Technology, Gothenburg, Sweden

Francesco Ambrosio - Computational Laboratory for Hybrid/Organic Photovoltaics (CLHYO), Istituto CNR di Scienze e Tecnologie Chimicie "Giulio Natta" (CNRSCITEC), 06123 Perugia, Italy; CNST@Polimi, Istituto Italiano di Tecnologia, Milano, Italy; (1) orcid.org/00000002-6388-9586

Filippo De Angelis - Department of Chemistry, Biology and Biotechnology, University of Perugia, 06123 Perugia, Italy; Computational Laboratory for Hybrid/Organic Photovoltaics (CLHYO), Istituto CNR di Scienze e Tecnologie Chimicie "Giulio Natta" (CNR-SCITEC), 06123 Perugia, Italy; CompuNet, Istituto Italiano di Tecnologia, 16163 Genova, Italy; $\odot$ orcid.org/0000-0003-3833-1975

Complete contact information is available at: https://pubs.acs.org/10.1021/acs.jpclett.1c01326

\section{Notes}

The authors declare no competing financial interest.

\section{ACKNOWLEDGMENTS}

The authors acknowledge funding from the "Area of Advance Materials Science" at Chalmers University of Technology and the Swedish Research Council (2019-03993). The computations were performed on resources provided by the Swedish National Infrastructure for Computing (SNIC) at NSC, C3SE, 
and PDC. This work was supported by Chalmers Gender Initiative for Excellence (Genie).

\section{REFERENCES}

(1) NREL. Best Research-Cell Efficiencies. https://www.nrel.gov/ pv/cell-efficiency.html (accessed 2021-03-29).

(2) Yang, W. S.; Noh, J. H.; Jeon, N. J.; Kim, Y. C.; Ryu, S.; Seo, J.; Seok, S. I. High-Performance Photovoltaic Perovskite Layers Fabricated Through Intramolecular Exchange. Science 2015, 348, 1234-1237.

(3) Saliba, M.; Matsui, T.; Domanski, K.; Seo, J.-Y.; Ummadisingu, A.; Zakeeruddin, S. M.; Correa-Baena, J.-P.; Tress, W. R.; Abate, A.; Hagfeldt, A.; et al. Incorporation of Rubidium Cations into Perovskite Solar Cells Improves Photovoltaic Performance. Science 2016, 354, 206-209.

(4) Yang, W. S.; Park, B.-W.; Jung, E. H.; Jeon, N. J.; Kim, Y. C.; Lee, D. U.; Shin, S. S.; Seo, J.; Kim, E. K.; Noh, J. H.; et al. Iodide Management in Formamidinium-Lead-Halide-Based Perovskite Layers for Efficient Solar Cells. Science 2017, 356, 1376-1379.

(5) Turren-Cruz, S.-H.; Hagfeldt, A.; Saliba, M. MethylammoniumFree, High-Performance, and Stable Perovskite Solar Cells on a Planar Architecture. Science 2018, 362, 449-453.

(6) Babayigit, A.; Ethirajan, A.; Muller, M.; Conings, B. Toxicity of Organometal Halide Perovskite Solar Cells. Nat. Mater. 2016, 15, 247-251.

(7) Stoumpos, C. C.; Malliakas, C. D.; Kanatzidis, M. G. Semiconducting Tin and Lead Iodide Perovskites with Organic Cations: Phase Transitions, High Mobilities, and Near-Infrared Photoluminescent Properties. Inorg. Chem. 2013, 52, 9019-9038.

(8) Green, M. A.; Ho-Baillie, A.; Snaith, H. J. The Emergence of Perovskite Solar Cells. Nat. Photonics 2014, 8, 506.

(9) Miyata, A.; Mitioglu, A.; Plochocka, P.; Portugall, O.; Wang, J. T.-W.; Stranks, S. D.; Snaith, H. J.; Nicholas, R. J. Direct Measurement of the Exciton Binding Energy and Effective Masses for Charge Carriers in Organic-Inorganic Tri-Halide Perovskites. Nat. Phys. 2015, 11, 582-587.

(10) Meggiolaro, D.; Ricciarelli, D.; Alasmari, A. A.; Alasmary, F. A.; De Angelis, F. Tin Versus Lead Redox Chemistry Modulates Charge Trapping and Self-Doping in Tin/lead Iodide Perovskites. J. Phys. Chem. Lett. 2020, 11, 3546-3556.

(11) Mahata, A.; Meggiolaro, D.; De Angelis, F. From Large to Small Polarons in Lead, Tin, and Mixed Lead-Tin Halide Perovskites. J. Phys. Chem. Lett. 2019, 10, 1790-1798.

(12) Ricciarelli, D.; Meggiolaro, D.; Ambrosio, F.; De Angelis, F. Instability of Tin Iodide Perovskites: Bulk P-Doping Versus Surface Tin Oxidation. ACS Energy Lett. 2020, 5, 2787-2795.

(13) Ambrosio, F.; Meggiolaro, D.; Almutairi, T. M.; De Angelis, F. Composition-Dependent Struggle Between Iodine and Tin Chemistry at the Surface of Mixed Tin/Lead Perovskites. ACS Energy Lett. 2021, 6, 969-976.

(14) Zhao, X.-G.; Dalpian, G. M.; Wang, Z.; Zunger, A. Polymorphous Nature of Cubic Halide Perovskites. Phys. Rev. B: Condens. Matter Mater. Phys. 2020, 101, 155137.

(15) Fabini, D. H.; Laurita, G.; Bechtel, J. S.; Stoumpos, C. C.; Evans, H. A.; Kontos, A. G.; Raptis, Y. S.; Falaras, P.; Van der Ven, A.; Kanatzidis, M. G.; et al. Dynamic Stereochemical Activity of the $\mathrm{Sn}^{2+}$ Lone Pair in Perovskite CsSnBr ${ }_{3}$. J. Am. Chem. Soc. 2016, 138, $11820-11832$.

(16) Wiktor, J.; Rothlisberger, U.; Pasquarello, A. Predictive Determination of Band Gaps of Inorganic Halide Perovskites. J. Phys. Chem. Lett. 2017, 8, 5507-5512.

(17) VandeVondele, J.; Krack, M.; Mohamed, F.; Parrinello, M.; Chassaing, T.; Hutter, J. Quickstep: Fast and Accurate Density Functional Calculations Using a Mixed Gaussian and Plane Waves Approach. Comput. Phys. Commun. 2005, 167, 103-128.

(18) Kühne, T. D.; et al. CP2K: An Electronic Structure and Molecular Dynamics Software Package-Quickstep: Efficient and Accurate Electronic Structure Calculations. J. Chem. Phys. 2020, 152, 194103.
(19) Perdew, J. P.; Ernzerhof, M.; Burke, K. Rationale for Mixing Exact Exchange with Density Functional Approximations. J. Chem. Phys. 1996, 105, 9982-9985.

(20) Bischoff, T.; Wiktor, J.; Chen, W.; Pasquarello, A. Nonempirical Hybrid Functionals for Band Gaps of Inorganic Metal-Halide Perovskites. Phys. Rev. Mater. 2019, 3, 123802.

(21) Österbacka, N.; Erhart, P.; Falletta, S.; Pasquarello, A.; Wiktor, J. Small Electron Polarons in $\mathrm{CsPbBr}_{3}$ : Competition Between Electron Localization and Delocalization. Chem. Mater. 2020, 32, 8393-8400.

(22) Kresse, G.; Hafner, J. Ab Initio Molecular Dynamics for Liquid Metals. Phys. Rev. B: Condens. Matter Mater. Phys. 1993, 47, 558.

(23) Kresse, G.; Furthmüller, J. Efficient Iterative Schemes for Ab Initio Total-Energy Calculations Using a Plane-Wave Basis Set. Phys. Rev. B: Condens. Matter Mater. Phys. 1996, 54, 11169.

(24) Ambrosio, F.; Mosconi, E.; Alasmari, A. A.; Alasmary, F. A.; Meggiolaro, D.; De Angelis, F. Formation of Color Centers in Lead Iodide Perovskites: Self-Trapping and Defects in the Bulk and Surfaces. Chem. Mater. 2020, 32, 6916-6924.

(25) Chen, Z.; Wang, J. J.; Ren, Y.; Yu, C.; Shum, K. Schottky Solar Cells Based on $\mathrm{CsSnI}_{3}$ Thin-Films. Appl. Phys. Lett. 2012, 101, 093901.

(26) Peedikakkandy, L.; Bhargava, P. Composition Dependent Optical, Structural and Photoluminescence Characteristics of Cesium Tin Halide Perovskites. RSC Adv. 2016, 6, 19857-19860.

(27) Zhou, L.; Katan, C.; Nie, W.; Tsai, H.; Pedesseau, L.; Crochet, J. J.; Even, J.; Mohite, A. D.; Tretiak, S.; Neukirch, A. J. Cation Alloying Delocalizes Polarons in Lead Halide Perovskites. J. Phys. Chem. Lett. 2019, 10, 3516-3524.

(28) Pisanu, A.; Speltini, A.; Quadrelli, P.; Drera, G.; Sangaletti, L.; Malavasi, L. Enhanced Air-Stability of Sn-Based Hybrid Perovskites Induced by Dimethylammonium (DMA): Synthesis, Characterization, Aging and Hydrogen Photogeneration of the $\mathrm{MA}_{1-X} \mathrm{DMA}_{x} \mathrm{SnBr}_{3}$ System. J. Mater. Chem. C 2019, 7, 7020-7026.

(29) Coduri, M.; Strobel, T. A.; Szafranski, M.; Katrusiak, A.; Mahata, A.; Cova, F.; Bonomi, S.; Mosconi, E.; De Angelis, F.; Malavasi, L. Band Gap Engineering in $\mathrm{MASnBr}_{3}$ and $\mathrm{CsSnBr}_{3}$ Perovskites: Mechanistic Insights Through the Application of Pressure. J. Phys. Chem. Lett. 2019, 10, 7398-7405.

(30) Zhang, Z.; Long, R.; Tokina, M. V.; Prezhdo, O. V. Interplay Between Localized and Free Charge Carriers Can Explain Hot Fluorescence in the $\mathrm{CH}_{3} \mathrm{NH}_{3} \mathrm{PbBr}_{3}$ Perovskite: Time-Domain $\mathrm{Ab}$ Initio Analysis. J. Am. Chem. Soc. 2017, 139, 17327-17333.

(31) Wiktor, J.; Ambrosio, F.; Pasquarello, A. Mechanism Suppressing Charge Recombination at Iodine Defects in $\mathrm{CH}_{3} \mathrm{NH}_{3} \mathrm{PbI}_{3}$ by Polaron Formation. J. Mater. Chem. A 2018, 6, 16863-16867.

(32) Ambrosio, F.; Wiktor, J.; De Angelis, F.; Pasquarello, A. Origin of Low Electron-Hole Recombination Rate in Metal Halide Perovskites. Energy Environ. Sci. 2018, 11, 101-105.

(33) Barrett, J.; Bird, S.; Donaldson, J.; Silver, J. The Mössbauer Effect in Tin (II) Compounds. Part XI. the Spectra of Cubic Trihalogenostannates (II). J. Chem. Soc. A 1971, 0, 3105-3108.

(34) Ferrara, C.; Patrini, M.; Pisanu, A.; Quadrelli, P.; Milanese, C.; Tealdi, C.; Malavasi, L. Wide Band-Gap Tuning in Sn-Based Hybrid Perovskites Through Cation Replacement: The $\mathrm{FA}_{1-X} \mathrm{MA}_{x} \mathrm{SnBr}_{3}$ Mixed System. J. Mater. Chem. A 2017, 5, 9391-9395.

(35) Pisanu, A.; Mahata, A.; Mosconi, E.; Patrini, M.; Quadrelli, P.; Milanese, C.; De Angelis, F.; Malavasi, L. Exploring the Limits of Three-Dimensional Perovskites: The Case of $\mathrm{FAPb}_{1-X} \mathrm{Sn}_{x} \mathrm{Br}_{3}$. ACS Energy Lett. 2018, 3, 1353-1359.

(36) Romani, L.; Speltini, A.; Ambrosio, F.; Mosconi, E.; Profumo, A.; Marelli, M.; Margadonna, S.; Milella, A.; Fracassi, F.; Listorti, A.; et al. Water-Stable $\mathrm{DMASnBr}_{3}$ Lead-Free Perovskite for Effective Solar-Driven Photocatalysis. Angew. Chem., Int. Ed. 2021, 60, 36113618. 